\title{
Timing of Surgery in Endocarditis Patient Presenting with Intracranial Haemorrhage, To Do or Not To Do?
}

\author{
Firas Aljanadi ${ }^{1 *}$, Amer Harky², Jun Heng Chong ${ }^{3}$ and Mark Pullan ${ }^{2}$ \\ ${ }^{1}$ Department of Cardiothoracic Surgery, Royal Victoria Hospital, UK \\ ${ }^{2}$ Department of Cardiothoracic Surgery, Liverpool Heart and Chest Hospital, UK \\ ${ }^{3}$ GKT School of Medical Education, King's College London, UK
}

\begin{abstract}
Introduction: Intracranial haemorrhage (ICH) is not uncommon as a complication of infective endocarditis (IE). However, the presence of intracranial haemorrhage would represent as a challenge to surgical intervention. Cardiac surgery intervention carries the potential risk of neurological deterioration perioperatively. Furthermore, the optimal timing of surgery Has been largly debated, and reports on operative outcomes for such cases are scarce.
\end{abstract}

Case presentation: Here we report a 41-year-old patient presented with neurologic symptoms caused by ICH secondary to multiple brain infarcts. Investigations led to the diagnosis of infective endocarditis which has later complicated with severe Aortic valve regurgitation (AR). Multi-disciplinary team decision was to proceed with urgent Aortic valve replacement. Early surgery within 48 hours was successful with no deterioration in neurological status.

Conclusion: Though Guidelines recommend delayed surgery in IE with $\mathrm{ICH}$, it is a luxury that we can't always afford. Early surgery when indicated can be performed safely without neurological deterioration. However, optimal timing should be considered on an individual basis.

\section{Keywords}

Infective endocarditis, Intracranial hemorrhage, Cerebrovascular complication, Aortic valve, Cardiac surgery, Aortic regurgitation

\section{Introduction}

Intracranial haemorrhage (ICH) is a known potential complication of infective endocarditis (IE) affecting around 5-7\% of cases $[1,2]$. Though surgery is indicated in severe aortic valve regurgitation due to endocarditis according to the ESC guidelines [3], that is not always plain sailing. Particularly in cases of endocarditis with intracranial haemorrhage where proceeding with cardiac surgery should be weighed against perioperative risks and postoperative prognosis. Furthermore, though surgery is indicated in acute aortic regurgitation (AR), the timing of surgery should be carefully addressed. While some authors advised against early surgery within seven days of onset [4], others advocated early surgical intervention [5].

We present our approach in a patient developed acute AR secondary to IE, who presented initially with neurological features due to $\mathrm{ICH}$ and multiple brain infarcts.

\section{Case Report}

A 41-year-old male presented to a local hospital with sudden onset of visual disturbance, acute headache, and left arm weakness. Apart from a recent history of recurrent fevers he is generally fit and well with no significant past medical history. Un urgent head CT scan (Figure 1) revealed a focus of acute intra-parenchymal bleed in the right parietal region $(19 \times 17 \mathrm{~mm})$ with an ischaemic wedge-shaped hypodensity in the right hemi-cerebellum. After neurosurgical referral, patient was managed conservatively for intracranial haemorrhage $(\mathrm{ICH})$ with plan to repeat scan next morning.

Brain CT angiogram the following day showed that the parietal haematoma increased in size $(22 \times 24 \mathrm{~mm})$. No convincing cause of $\mathrm{ICH}$ (aneurysm or vascular malformation neither in anterior nor in posterior circulation) was observed and the

*Corresponding author: Firas Aljanadi, Department of Cardiothoracic Surgery, Royal Victoria Hospital, 274 Grosvenor Rd, BT12 6BA, Belfast, UK

Accepted: December 12, 2020

Published online: December 14, 2020

Citation: Aljanadi F, Harky A, Chong JH, et al. (2020) Timing of Surgery in Endocarditis Patient Presenting with Intracranial Haemorrhage, To Do or Not To Do?. J Cardiothorac Surg Ther 4(1):68-71 
Citation: Aljanadi F, Harky A, Chong JH, et al. (2020) Timing of Surgery in Endocarditis Patient Presenting with Intracranial Haemorrhage, To Do or Not To Do?. J Cardiothorac Surg Ther 4(1):68-71

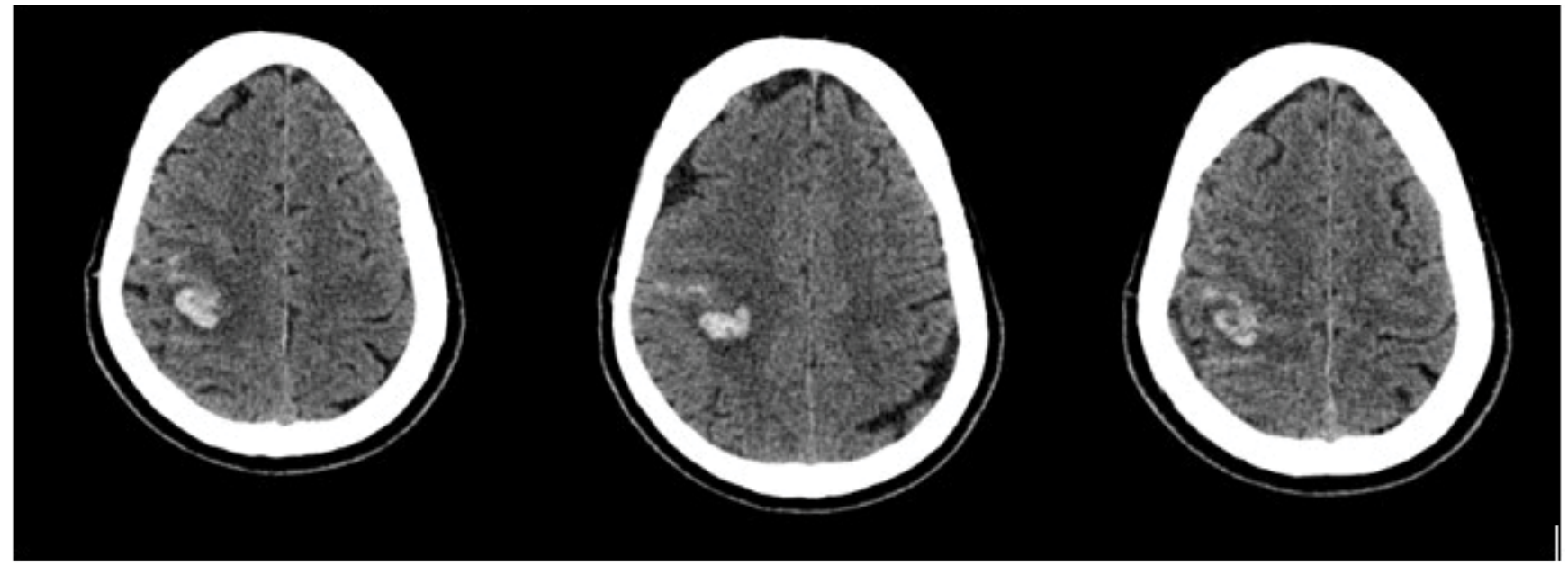

Figure 1: CT Head (axial views) shows $19 \times 17 \mathrm{~mm}$ focus of acute intraparenchymal bleed seen in the right high parietal region.

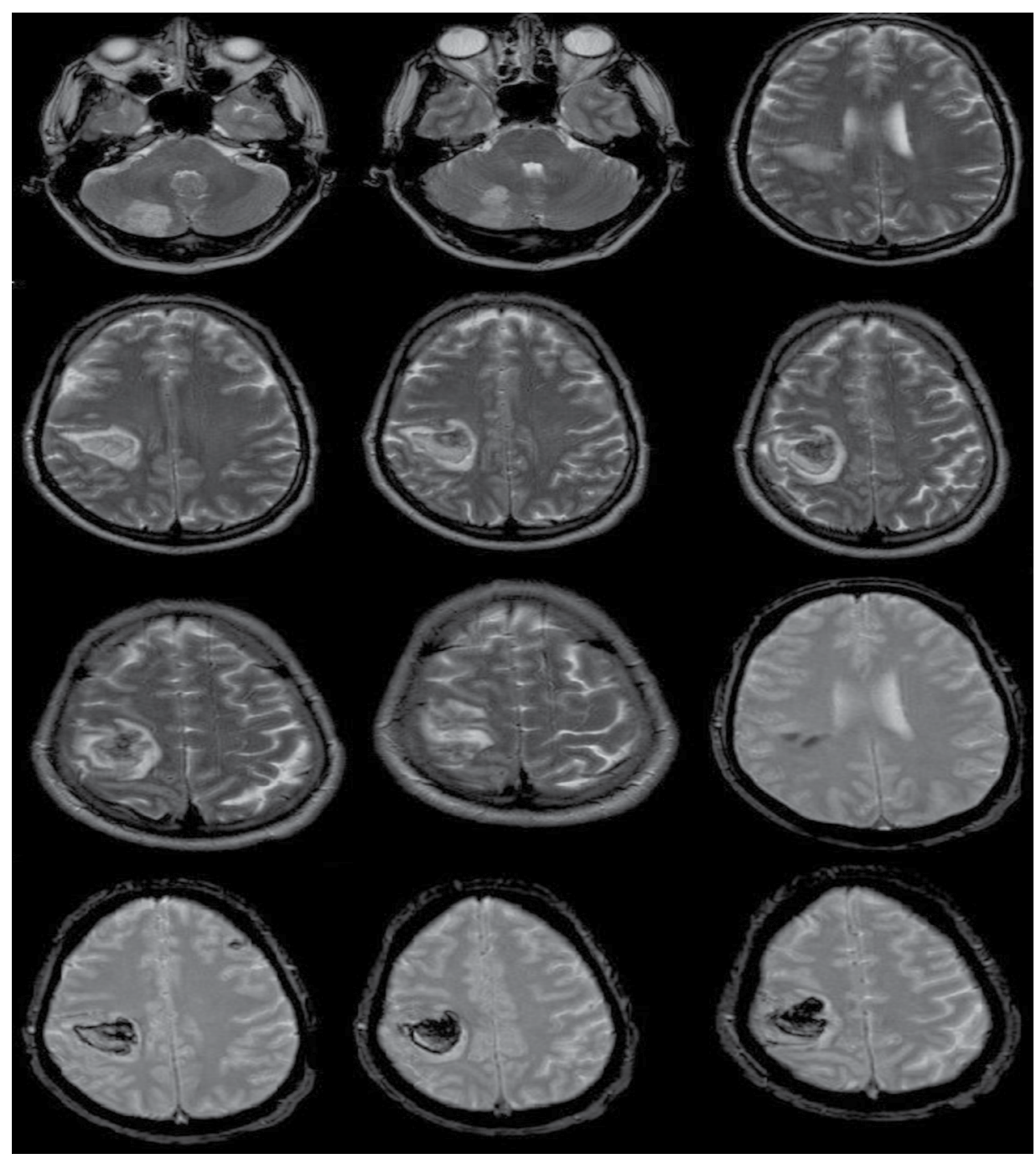

Figure 2: Brain MRI showing multiple acute infarcts - with large haemorrhagic infarct in the right parital region and acute infarction of the splenium of the corpus callosum.

main cause of this acute bleed remained unclear. Clinically, apart from slight shortness of breath (SOB), the patient haemodynamically was still stable without medical support, with
Oxygen saturation 99\%. Looking for the reason of $\mathrm{ICH}$, later on the day, a head MRI was done (Figure 2). This showed multiple acute infarcts including a large haemorrhagic infarct in the 
Citation: Aljanadi F, Harky A, Chong JH, et al. (2020) Timing of Surgery in Endocarditis Patient Presenting with Intracranial Haemorrhage, To Do or Not To Do?. J Cardiothorac Surg Ther 4(1):68-71

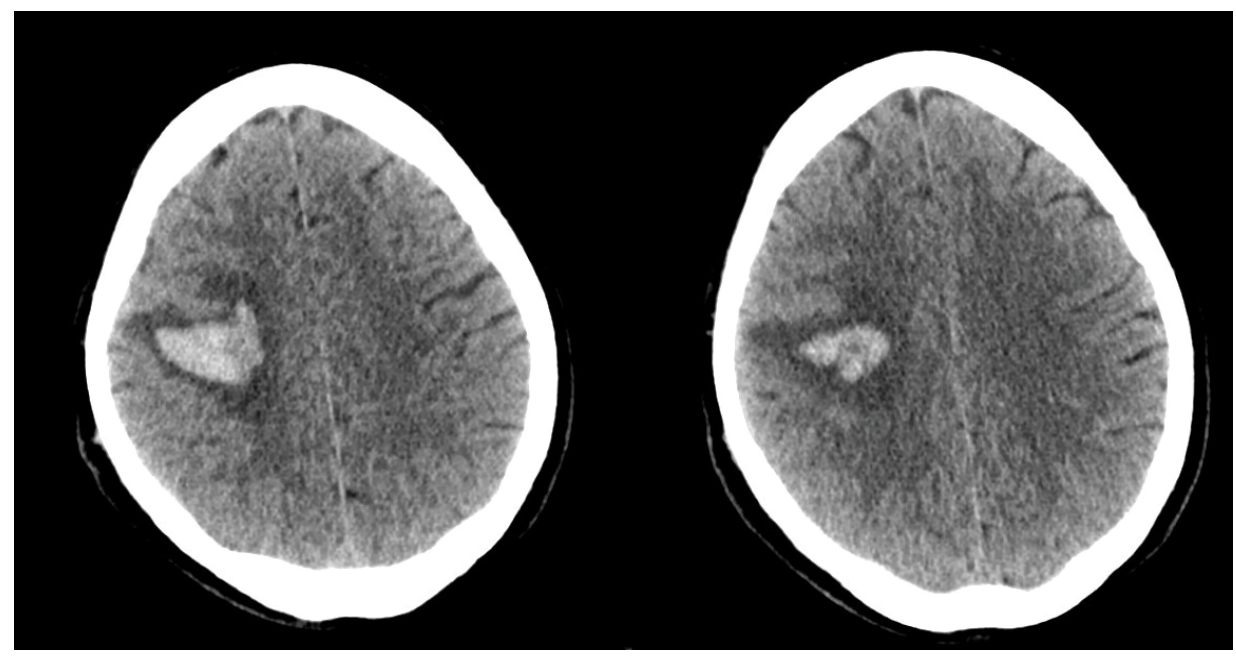

Figure 3: CT Head postoperatively with no change in the size of acute intraparenchymal haematoma in the right fronto-parietal area with surrounding oedema as before.

right parital region and acute infarction of the splenium of the corpus callosum. Due to these multiple infarcts and in view of the history of fever and SOB, a transthoracic echocardiography (TTE) was done and revealed severe AR and possible vegetation. SOB was getting worse and Oxygen requirement was increasing requiring transfer to our hospital intensive care unit. Transoesophageal echo (TOE) confirmed an aortic vegetations involving all 3 cusps, severe AR with maintained left ventricular systolic function. Chest $\&$ abdomen CT scan showed bilateral pleural effusion, with multiple splenic and hepatic infarcts.

Though it is now ascertained that his $\mathrm{ICH}$ and brain infarcts are secondary to aortic valve IE, the source of endocarditis is yet unknown. He had a history of swollen right shoulder recently, with unsuccessful attempt of aspiration by rheumatologist. His dentition is good, attending dentist regularly. Blood cultures indicated methicillin-susceptible Staphylococcus aureus (MSSA) as the causative agent of endocarditis. He was started on flucloxacillin and gentamicin which was replaced later with ciprofloxacin. Although he was alert oriented with the same degree of left arm weakness, he started to become gradually tachycardic, tachypnoeic unable to complete a full sentence with further increasing Oxygen requirements. Chest X-ray showed features of pulmonary oedema. After a multi-disciplinary meeting, it was advisable to proceed with the surgery at the earliest opportunity.

In Theatre, after Cardio-Pulmonary bypass (CPB) was established. Active coagulation time (ACT) was regularly monitored and kept to a minimum to reduce the risk of $\mathrm{ICH}$ deterioration. We aimed as possible for short CPB time (58 minutes) and aortic cross clamp time (51 minutes). With $\mathrm{CO}_{2}$ diffusion and under mild hypothermia $\left(34 \mathrm{~b}^{\circ} \mathrm{C}\right)$, the ascending aorta and right atrium were cannulated. Retrograde (cold blood) cardioplegic arrest was achieved after aortic cross clamp was applied. Intra-operative findings included very dilated heart, torrential $A R$, and no mitral valve lesions. The aortic valve was excised and replaced with Trifecta bioprosthetic $25 \mathrm{~mm}$ valve. CPB weaning was facilitated with temporary epicardial pacing and low-dose noradrenaline. TOE showed a well-seated aortic valve with no paravalvular leak.

Postoperative recovery in the ICU was uneventful. Head CT (Figure 3) showed stable haemorrhagic infarct in the right fronto-parietal region and ischaemia in right cerebellar region. No new features. He was continued on IV diuretics and high flow $\mathrm{O}_{2}$ to help to resolve the pulmonary oedema. Despite reduced power in left arm, he was fully oriented without new neurologic deficits. He was discharged to ward after two days in ITU when oxygen requirement was reduced and pulmonary oedema had improved.

He progressed well on IV antibiotics and physiotherapy until he was transferred to a neurology rehabilitation unit with the residual left arm weakness on the $15^{\text {th }}$ postoperative day with a plan to continue antibiotics and follow up in clinic in 6 weeks.

\section{Discussion}

Symptomatic neurological complications in infective endocarditis patients occur mainly due to embolization of vegetations. These complications happen in $15-30 \%$ of patients with IE [3]. In particular, ICH complicates $5-7 \%$ of cases of IE and it is a common complication of left-sided IE [1,2]. ICH is associated with a significantly higher risk of surgical mortality [6].

Multi-disciplinary meeting (MDT) discussion, in our case, enhanced significantly the process of decision-making by involving the neurologist who was looking after him, the anaesthetist, the intensivist and other surgical colleagues. In addition to that, repeating the head CT scan did not show any interval changes in the ICH appearance. The patient had Staphylococcus aureus endocarditis causing haemorrhagic parietal CVA and other multiple ischaemic lesions, and abdomen CT scan showed evidence of embolic infarcts in spleen and liver. Additionally, in making our decision we found the National Institutes of Health Stroke Scale (NIHSS) score very helpful. The NIHSS has been repetitively authorised as a tool for evaluating stroke severity and as an excellent predictor for 
Citation: Aljanadi F, Harky A, Chong JH, et al. (2020) Timing of Surgery in Endocarditis Patient Presenting with Intracranial Haemorrhage, To Do or Not To Do?. J Cardiothorac Surg Ther 4(1):68-71

patient outcomes [7]. His low NIHSS score $(=4)$ would suggest that the best option would be to have his aortic valve operated on early. The heart has become increasingly decompensated which made surgery the most appropriate management though the potential risk of deteriorated ICH due to the anticoagulation status required while patient is on $\mathrm{CPB}$.

The patient was involved in decision making and made aware of different consequences that might result from this open-heart surgery. He was aware that there is a risk of his stroke worsening if he has aortic valve replacement (AVR) but waiting would cause his demise through heart failure. In terms of valve choices, we believed he would better have a tissue valve as anticoagulating him could create further deterioration though a second time AVR could be carried out in future with higher than usual risks of morbidity and mortality.

The existence of ICH with IE challenges the surgical judgment. According to the Japanese Adult Cardiovascular Surgery Database, patients with IE and cerebral haemorrhage showed trends toward lower incidence of hospital death when operated on between 1-3 weeks than those operated on within 7 days [4]. Furthermore, the European Society of Cardiology (ESC) guidelines recommend in IE patients with intracranial hemorrhage that surgery should generally be postponed for $\geq$ 1 month (Class Ila; Level of Evidence B) [3].

The timing of surgery in IE complicated with $\mathrm{ICH}$ is a debatable topic [8]. In review of medical literature, Okita, et al. found that early surgical treatment is safe in active IE patients with cerebral infarction. However, surgery within 7 days should be avoided in patients with ICH [4]. Carell [5], in his comment on this study, although he was arguing that in patients with IE and CVA with or without ICH early valve surgery is not generally contraindicated, he fully accepted the conclusions of Okita, et al. [4].

We believed that individual assessment of each case is essential in decision making and planning surgery. Surgery could be justifiable in cases where emergency surgery is indicated, and the ICH is small. Others insisted that when emergent or urgent surgery is required for IE, neurological complications should not be a cause of deferral or delay [9]. In a series of 30 patients with ICH secondary to IE, Yoshioka, et al. [10] reported 20 of them had valve surgery within 28 days. Neither neurologic deterioration nor exacerbation of haemorrhagic lesions occurred in all these patients irrespective of the timing of surgery as the post-operative neuroimaging study revealed [10].

\section{Conclusion}

Early surgical intervention in IE complicated by $\mathrm{ICH}$ can be lifesaving with low risk of neurological events. A thorough neurovascular imaging studies can help to identify and assess patient's risk factors. Decision and Timing of surgery should be judicious and individual when endocarditis presents with intracranial haemorrhage and severe aortic regurgitation.

\section{Declarations}

\section{Conflict of interest}

The authors certify that there is no conflict of interest with any financial organization regarding the material discussed in the manuscript.

\section{Funding}

The authors report no involvement in the research by the sponsor that could have influenced the outcome of this work.

\section{References}

1. Derex L, Bonnefoy E, Delahaye F (2010) Impact of stroke on therapeutic decision making in infective endocarditis. J Neurol 257: 315-321.

2. Salaun E, Touil A, Hubert $S$, et al. (2018) Intracranial haemorrhage in infective endocarditis. Arch Cardiovasc Dis 111: 712721.

3. Gilbert Habib, Patrizio Lancellotti, Manuel J Antunes, et al. (2015) 2015 ESC Guidelines for the management of infective endocarditis. European Heart Journal 36: 3075-3128.

4. Okita Y, Minakata K, Yasuno S, et al. (2016) Optimal timing of surgery for active infective endocarditis with cerebral complications: A Japanese multicentre study. Eur J Cardiothorac Surg 50: 374-382.

5. Carrel T (2016) Early valve repair or replacement is not generally contraindicated in patients with infective endocarditis and stroke with or without intracranial haemorrhage. Eur J Cardiothorac Surg 50: 383-384.

6. Diab M, Guenther A, Scheffel P, et al. (2016) Can radiological characteristics of preoperative cerebral lesions predict postoperative intracranial haemorrhage in endocarditis patients? Eur J Cardiothorac Surg 49: e119-e126.

7. Frankel MR, Morgenstern LB, Kwiatkowski T, et al. (2000) Predicting prognosis after stroke: a placebo group analysis from the National Institute of Neurological Disorders and Stroke rt-PA Stroke Trial. Neurology 55: 952-959.

8. Yong X, Weiliang Z, Yili C, et al. (2015) A case of successful treatment of infective endocarditis complicated with intracranial hemorrhage, and literature review. Heart Surg Forum 18: e198-e200.

9. Wan S, Sung K, Park PW, et al. (2014) Stroke is not a treatment dilemma for early valve surgery in active infective endocarditis. J Heart Valve Dis 23: 609-616.

10. Yoshioka D, Toda K, Sakaguchi T, et al. (2014) Valve surgery in active endocarditis patients complicated by intracranial haemorrhage: The influence of the timing of surgery on neurological outcomes. Eur J Cardiothorac Surg 45: 1082-1088.

DOI: $10.36959 / 582 / 427$

Copyright: (C) 2020 Aljanadi F, et al. This is an open-access article distributed under the terms of the Creative Commons Attribution License, which permits unrestricted use, distribution, and reproduction in any medium, provided the original author and source are credited. 